\title{
NUMERICAL RESULTS OF MIXED CONVECTION FLOW OVER A FLAT PLATE WITH THE IMPOSED HEAT AND ANGLE OF INCLINATION
}

\author{
Mohammad Ghani ${ }^{1 *}$ \\ ${ }^{1}$ Faculty of Advanced Technology and Multidiscipline, Airlangga University, Surabaya 60115, Indonesia \\ *Corresponding Author.Email: mohammad.ghani2013@gmail.com
}

\begin{abstract}
In this paper, the numerical results of mixed convection flow over a flat plate with the imposed heat and different angles of inclination are established by applying the finite difference method of Crank-Nicolson. We further compare these numerical results with the case of non-mixed convection flow. The velocity and temperature profiles are decreased when the different values of the Prandtl number $\left(P_{r}\right)$ are increased. Meanwhile, the velocity profiles are increased, when the different values of angle of inclination $(\alpha)$ and mixed convection parameter $(\lambda)$ are increased. The mixed convection flow case $(\lambda=1.5)$ is affected by the external force, so the velocity of convection flow is higher than the non-mixed case $(\lambda=0)$.
\end{abstract}

Keywords: Mixed Convection Flow; Angle of Inclination; Crank-Nicolson Method

\section{Introduction}

The numerical results of mixed convection flow over a flat plate with the imposed heat have been extensively studied by researchers and widely applied in engineering applications. Many researchers investigated the cases of mixed convection flow in different geometries such as sphere, cone, and cylinder with fluids Newtonian or non-Newtonian. Amin et al [1] studied the steady and incompressible mixed convection flow over a sphere with the constant temperature which was solved numerically by Keller-Box. Nazar et al [2] investigated the mixed convection flow over a sphere with Newtonian heating which was also established by Keller-Box method. The Newtonian heating was proportional to the local surface temperature. Kasim [3] studied the steady-state and incompressible mixed convection flow of viscoelastic fluid over a sphere that was established numerically by KellerBox method. Ghani et al [4] studied the steady-state and incompressible mixed convection over sphere, where the Crank-Nicolson and iterative method are employed in numerical results. Moreover, Ghani and Wayan [5] studied the previous mathematical model by imposing the effect of MHD, where Keller-Box method was applied to establish the numerical results. Widodo et al [6] studied the unsteady-state boundary layer MHD of micro-polar fluid over a solid sphere.

Recently, the studies of ferrofluid included the study of heat, thermal radiation and slip flow of ferrofluid towards various geometry like stagnation point, stretching/shrinking surface as well as a flat surface with heat flux and Newtonian heating boundary conditions by Khan et al., [7], Zeeshan et al., [8], Jusoh et al., [9], Mohamed et al., [10], Yasin et al., [11], Gangadhar et al., [12] and Kumar et al., [13]. For the case of industrial segment, the ferrofluid is applied in electronic devices cooling system for example in hi-fi speakers and computer hard-disc. Moreover, the ferrofluid was also employed in medical treatment segment. The ferrofluid play role in cancer therapy, bleeding stopping agent, magnetic resonance imaging and other diagnostic tests [14]. Studying the ferrofluid potential in medicine, specifically blood as the based-fluid is challenging. The blood is a non-Newtonian fluid, because it has the different characteristics with a Newtonian fluid of water. The ordinary Navier- 
Stokes equations did not represents the characteristics of the non-Newtonian fluid model. Then, we need to modify the Navier-Stokes equations to handle the non-Newtonian fluid model [15]. Casson model is introduced as the part of the non-Newtonian fluid model to characterize the elastic solid of fluid behaviour.

Based on the last studies, we present the numerical results of mixed convection flow over a flat plate with the imposed heat and angle of inclination, where this numerical solution is solved by finite difference method of Crank-Nicolson.

\section{Mathematical Model}

We first present the following two-dimensional mathematical model of mixed convection flow over a flat plate with the imposed heat and angle of inclination $(\alpha)$

$$
\begin{gathered}
\frac{\partial \bar{\psi}}{\partial \bar{x}}+\frac{\partial \bar{\varphi}}{\partial \bar{y}}=0 \\
\frac{\partial \bar{\psi}}{\partial \bar{t}}+\bar{\psi} \frac{\partial \bar{\psi}}{\partial \bar{x}}+\bar{\varphi} \frac{\partial \bar{\psi}}{\partial \bar{y}}=\nu \frac{\partial^{2} \bar{\psi}}{\partial \bar{y}^{2}}+g_{0} \beta\left(\bar{T}-T_{\infty}\right) \sin (\alpha), \\
\frac{\partial \bar{T}}{\partial \bar{t}}+\bar{\psi} \frac{\partial \bar{T}}{\partial \bar{x}}+\bar{\varphi} \frac{\partial \bar{T}}{\partial \bar{y}}=\frac{k}{\rho c_{p}} \frac{\partial^{2} \bar{T}}{\partial \bar{y}^{2}}
\end{gathered}
$$

with the initial and boundary conditions

for $\bar{t} \leq 0$,

$$
\bar{\psi}=0, \bar{\varphi}=0, \bar{T}=T_{\infty},
$$

for $\bar{t}>0$

$$
\begin{aligned}
& \bar{\psi}=0, \bar{\varphi}=0, \bar{T}=T_{\infty}, \text { at } \bar{x}=0 \\
& \bar{\psi}=0, \bar{\varphi}=0, \bar{T}=T_{w}, \text { at } \bar{y}=0 \\
& \bar{\psi} \rightarrow 0, \bar{\varphi} \rightarrow 0, \bar{T} \rightarrow T_{\infty}, \text { as } \bar{y} \rightarrow \infty
\end{aligned}
$$

We further transform the non-dimensional mathematical model, boundary, and initial conditions (1)-(5) by applying the following non-dimensional variables

$$
x=\frac{\bar{x}}{L}, y=\frac{\bar{y}}{L}, \psi=\frac{\bar{\psi} L}{v}, \varphi=\frac{\bar{\varphi} L}{v}, \theta=R_{e}^{2}\left(\frac{\bar{T}-T_{\infty}}{T_{w}-T_{\infty}}\right)
$$

where $L, R_{e}$, and $\theta$ are characteristic of length of plate, Reynold number, and non-dimensional temperature respectively. The presence of Reynold number $R_{e}$ is due to the mixed convection flow. The mixed convection flow is the combination between natural (Grashof number, $G_{r}$ ) and forced (Reynold number, $R_{e}$ ) convection flow, where this correlation can be stated as $G_{r} / R_{e}^{2}$. If $G_{r} \cong R_{e}^{2}$ then the natural and convection flow are significant, if $R_{e}^{2} \ll G_{r}$ then the natural convection flow is only significant, and if $G_{r} \ll R_{e}^{2}$ then the forced convection flow is only significant. By applying the non-dimensional variables (6) to (1)-(5), then the following non-dimensional equations are obtained

$$
\begin{gathered}
\frac{\partial \psi}{\partial x}+\frac{\partial \varphi}{\partial y}=0 \\
\frac{\partial \psi}{\partial t}+\psi \frac{\partial \psi}{\partial x}+\varphi \frac{\partial \psi}{\partial y}=v \frac{\partial^{2} \psi}{\partial y^{2}}+\lambda \theta \sin (\alpha)
\end{gathered}
$$




$$
\frac{\partial \theta}{\partial t}+\psi \frac{\partial \theta}{\partial x}+\varphi \frac{\partial \theta}{\partial y}=\frac{1}{P_{r}} \frac{\partial^{2} \theta}{\partial y^{2}}
$$

with the initial and boundary conditions

for $t \leq 0$,

$$
\psi=0, \varphi=0, \theta=0,
$$

for $t>0$

$$
\begin{aligned}
& \psi=0, \varphi=0, \theta=0, \text { at } x=0 \\
& \psi=0, \varphi=0, \theta=0, \text { at } y=0 \\
& \psi \rightarrow 0, \varphi \rightarrow 0, \theta \rightarrow 0, \text { as } y \rightarrow \infty
\end{aligned}
$$

where $\lambda=\frac{G_{r}}{R_{e}^{2}}$ is mixed convection parameter, $P_{r}=\frac{v \rho c_{p}}{R_{e}^{2} k}$ is Prandtl number, and $G_{r}=\frac{g_{0} \beta L^{3}\left(T_{w}-T_{\infty}\right)}{v^{2}}$ is Grashof number.

\section{Numerical Analysis}
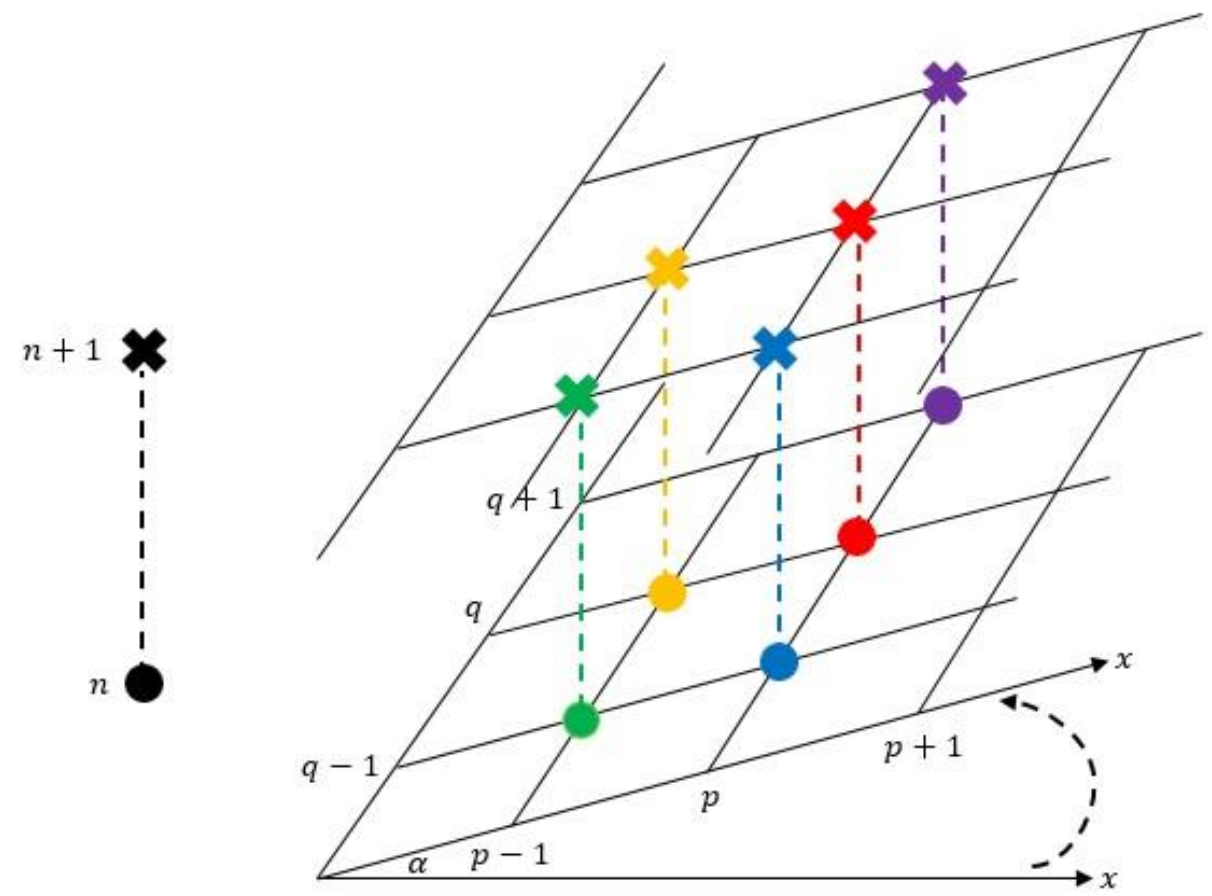

Figure 1. Stencil of Crank-Nicolson method

Figure 1 represents the stencil of Crank-Nicolson method, where $\alpha$ is angle of inclination from axis- $x$. The indexes of $p, q$, and $n$ are respectively representation of axis- $x$, axis- $y$, and time- $t$. We further apply the Crank-Nicolson method to non-dimensional equations (7)-(9), then we have the following discretization results

$$
\begin{aligned}
& \frac{\left(\psi_{p, q}^{n+1}-\psi_{p-1, q}^{n+1}\right)+\left(\psi_{p, q}^{n}-\psi_{p-1, q}^{n}\right)+\left(\psi_{p, q-1}^{n+1}-\psi_{p-1, q-1}^{n+1}\right)+\left(\psi_{p, q-1}^{n}-\psi_{p-1, q-1}^{n}\right)}{2 \Delta x}+ \\
& \frac{\left(\varphi_{p, q}^{n+1}-\varphi_{p, q-1}^{n+1}\right)+\left(\varphi_{p, q}^{n}-\varphi_{p, q-1}^{n}\right)}{\Delta y}=0
\end{aligned}
$$




$$
\begin{aligned}
& \frac{\psi_{p, q}^{n+1}-\psi_{p, q}^{n}}{\Delta t}+\psi_{p, q}^{n} \frac{\left(\psi_{p, q}^{n+1}-\psi_{p-1, q}^{n+1}\right)+\left(\psi_{p, q}^{n}-\psi_{p-1, q}^{n}\right)}{2 \Delta x}+ \\
& \varphi_{p, q}^{n} \frac{\left(\psi_{p, q+1}^{n+1}-\psi_{p, q-1}^{n+1}\right)+\left(\psi_{p, q+1}^{n}-\psi_{p, q-1}^{n}\right)}{4 \Delta y}=\frac{\theta_{p, q}^{n+1}+\theta_{p, q}^{n}}{2} \lambda \sin (\alpha)+ \\
& \frac{\left(\psi_{p, q-1}^{n+1}-2 \psi_{p, q}^{n+1}+\psi_{p, q+1}^{n+1}\right)+\left(\psi_{p, q-1}^{n}-2 \psi_{p, q}^{n}+\psi_{p, q+1}^{n}\right)}{2 \Delta y^{2}} \\
& \frac{\theta_{p, q}^{n+1}-\theta_{p, q}^{n}}{\Delta t}+\psi_{p, q}^{n} \frac{\left(\theta_{p, q}^{n+1}-\theta_{p-1, q}^{n+1}\right)+\left(\theta_{p, q}^{n}-\theta_{p-1, q}^{n}\right)}{2 \Delta x}+ \\
& \varphi_{p, q}^{n} \frac{\left(\theta_{p, q+1}^{n+1}-\theta_{p, q-1}^{n+1}\right)+\left(\theta_{p, q+1}^{n}-\theta_{p, q-1}^{n}\right)}{4 \Delta y}= \\
& \frac{1}{P_{r}} \frac{\left(\theta_{p, q-1}^{n+1}-2 \theta_{p, q}^{n+1}+\theta_{p, q+1}^{n+1}\right)+\left(\theta_{p, q-1}^{n}-2 \theta_{p, q}^{n}+\theta_{p, q+1}^{n}\right)}{2 \Delta y^{2}}
\end{aligned}
$$

with the initial and boundary conditions

for $n=0$,

$$
\psi_{p, q}^{0}=0, \varphi_{p, q}^{0}=0, \theta_{p, q}^{0}=0,
$$

for $n=1,2,3, \ldots, N_{t}$

$$
\begin{aligned}
& \psi_{0, q}^{n}=0, \varphi_{0, q}^{n}=0, \theta_{0, q}^{n}=0, \text { at } p=0 \\
& \psi_{p, 0}^{n}=0, \varphi_{p, 0}^{n}=0, \theta_{p, 0}^{n}=1, \text { at } q=0 \\
& \psi_{p, N_{y}}^{n}=0, \varphi_{p, N_{y}}^{n}=0, \theta_{p, N_{y}}^{n}=0, \text { at } q=N_{y}
\end{aligned}
$$

Rearranging (12)-(14) and grouping the same indexes $n+1$ and $n$, then we have have the following new results of discretization

$$
\begin{aligned}
& \varphi_{p, q}^{n+1}=\varphi_{p, q-1}^{n+1}-\varphi_{p, q}^{n}+\varphi_{p, q-1}^{n}- \\
& t_{1}\left(\psi_{p, q}^{n+1}-\psi_{p-1, q}^{n+1}+\psi_{p, q}^{n}-\psi_{p-1, q}^{n}+\psi_{p, q-1}^{n+1}-\psi_{p-1, q-1}^{n+1}+\psi_{p, q-1}^{n}-\psi_{p-1, q-1}^{n}\right) \\
& \left(-s_{2}-s_{3}\right) \psi_{p, q-1}^{n+1}+\left(1+s_{1}+2 s_{3}\right) \psi_{p, q}^{n+1}+\left(s_{2}-s_{3}\right) \psi_{p, q+1}^{n+1}= \\
& \psi_{p, q}^{n}+s_{1}\left(\psi_{p-1, q}^{n+1}+\psi_{p-1, q}^{n+}-\psi_{p, q}^{n}\right)+s_{2}\left(\psi_{p, q-1}^{n}-\psi_{p, q+1}^{n}\right)+ \\
& s_{3}\left(\psi_{p, q-1}^{n}-2 \psi_{p, q}^{n}+\psi_{p, q+1}^{n}\right)+s_{4}\left(\theta_{p, q}^{n}+\theta_{p, q}^{n}\right) \\
& \left(-r_{2}-r_{3}\right) \theta_{p, q-1}^{n+1}+\left(1+r_{1}+2 r_{3}\right) \theta_{p, q}^{n+1}+\left(r_{2}-r_{3}\right) \theta_{p, q+1}^{n+1}= \\
& \theta_{p, q}^{n}+r_{1}\left(\theta_{p-1, q}^{n+1}+\theta_{p-1, q}^{n}-\theta_{p, q}^{n}\right)+r_{2}\left(\theta_{p, q-1}^{n}-\theta_{p, q+1}^{n}\right)+r_{3}\left(\theta_{p, q-1}^{n}-2 \theta_{p, q}^{n}+\theta_{p, q+1}^{n}\right)
\end{aligned}
$$

where 


$$
\begin{aligned}
& r_{1}=s_{1}=\frac{\Delta t \psi_{p, q}^{n}}{2 \Delta x}, t_{1}=\frac{\Delta y}{2 \Delta x}, r_{2}=s_{2}=\frac{\Delta t \varphi_{p, q}^{n}}{4 \Delta y}, \\
& r_{3}=\frac{\Delta t}{P_{r} 2 \Delta y^{2}}, s_{3}=\frac{\Delta t}{2 \Delta y^{2}}, s_{4}=\left(\frac{\Delta t}{2}\right) \lambda \sin (\alpha)
\end{aligned}
$$

We further bring all discretization results (17)-(19) into the following tri-diagonal matrices

$$
\begin{array}{r}
A_{1} \theta_{p, q-1}^{n+1}+B_{1} \theta_{p, q}^{n+1}+C_{1} \theta_{p, q+1}^{n+1}=E_{1} \\
A_{2} \psi_{p, q-1}^{n+1}+B_{2} \psi_{p, q}^{n+1}+C_{2} \psi_{p, q+1}^{n+1}=E_{2} \\
\varphi_{p, q}^{n+1}=E_{3}
\end{array}
$$

where

$$
\begin{aligned}
A_{1}= & -r_{2}-r_{3}, B_{1}=1+r_{1}+2 r_{3}, C_{1}=r_{2}-r_{3} \\
A_{2}= & -s_{2}-s_{3}, B_{2}=1+s_{1}+2 s_{3}, C_{2}=s_{2}-s_{3} \\
E_{1}= & \theta_{p, q}^{n}+r_{1}\left(\theta_{p-1, q}^{n+1}+\theta_{p-1, q}^{n}-\theta_{p, q}^{n}\right)+r_{2}\left(\theta_{p, q-1}^{n}-\theta_{p, q+1}^{n}\right)+r_{3}\left(\theta_{p, q-1}^{n}-2 \theta_{p, q}^{n}+\theta_{p, q+1}^{n}\right) \\
E_{2}= & \psi_{p, q}^{n}+s_{1}\left(\psi_{p-1, q}^{n+1}+\psi_{p-1, q}^{n}-\psi_{p, q}^{n}\right)+s_{2}\left(\psi_{p, q-1}^{n}-\psi_{p, q+1}^{n}\right)+ \\
& s_{3}\left(\psi_{i, j-1}^{n}-2 \psi_{i, j}^{n}+\psi_{i, j+1}^{n}\right)+s_{4}\left(\theta_{i, j}^{n+1}+\theta_{i, j}^{n}\right) \\
E_{3}= & \varphi_{p, q-1}^{n+1}-\varphi_{p, q}^{n}+\varphi_{p, q-1}^{n}-
\end{aligned}
$$$$
t_{1}\left(\psi_{p, q}^{n+1}-\psi_{p-1, q}^{n+1}+\psi_{p, q}^{n}-\psi_{p-1, q}^{n}+\psi_{p, q-1}^{n+1}-\psi_{p-1, q-1}^{n+1}+\psi_{p, q-1}^{n}-\psi_{p-1, q-1}^{n}\right)
$$

Furthermore, the discretization of (21) and (22) can be represented into tri-diagonal matrices to make it easy in solving iteratively with the Thomas algorithm. This process is only subscript $q$ to be iterated, that is $q=1,2,3, \ldots, N_{y}-2, N_{y}-1$.

Then, by assuming $\left[U_{p, 1}^{n+1}, U_{p, 2}^{n+1}, \ldots, U_{p, N_{y}-2}^{n+1}, U_{p, N_{y}-1}^{n+1}\right]=\left[\psi_{p, 1}^{n+1}, \psi_{p, 2}^{n+1}, \ldots, \psi_{p, N_{y}-2}^{n+1}, \psi_{p, N_{y}-1}^{n+1}\right]$, we can derive the following matrix

$$
\begin{gathered}
{\left[\begin{array}{cccccc}
B_{1} & C_{1} & 0 & 0 & \ldots & 0 \\
A_{1} & B_{1} & C_{1} & 0 & \ldots & 0 \\
0 & A_{1} & B_{1} & C_{1} & \ldots & \vdots \\
0 & 0 & \ddots & \ddots & \ddots & 0 \\
\vdots & \vdots & \vdots & A_{1} & B_{1} & C_{1} \\
0 & 0 & \ldots & 0 & A_{1} & B_{1}
\end{array}\right]\left[\begin{array}{c}
\theta_{p, 1}^{n+1} \\
\theta_{p, 2}^{n+1} \\
\theta_{p, 3}^{n+1} \\
\vdots \\
\theta_{p, N_{y}-2}^{n+1} \\
\theta_{p, N_{y}-1}^{n+1}
\end{array}\right]=\left[\begin{array}{c}
E_{1} \\
E_{1} \\
E_{1} \\
\vdots \\
E_{1} \\
E_{1}
\end{array}\right]+\left[\begin{array}{c}
\theta_{p, 0}^{n+1} \\
0 \\
0 \\
\vdots \\
0 \\
\theta_{p, N_{y}}^{n+1}
\end{array}\right]} \\
{\left[\begin{array}{cccccc}
B_{2} & C_{2} & 0 & 0 & \ldots & 0 \\
A_{2} & B_{2} & C_{2} & 0 & \ldots & 0 \\
0 & A_{2} & B_{2} & C_{2} & \ldots & \vdots \\
0 & 0 & \ddots & \ddots & \ddots & 0 \\
\vdots & \vdots & \vdots & A_{2} & B_{2} & C_{2} \\
0 & 0 & \ldots & 0 & A_{2} & B_{2}
\end{array}\right]\left[\begin{array}{c}
U_{p, 1}^{n+1} \\
U_{p, 2}^{n+1} \\
U_{p, 3}^{n+1} \\
\vdots \\
U_{p, N_{y}-2}^{n+1} \\
U_{p, N_{y}-1}^{n+1}
\end{array}\right]=\left[\begin{array}{c}
E_{2} \\
E_{2} \\
E_{2} \\
\vdots \\
E_{2} \\
E_{2}
\end{array}\right]+\left[\begin{array}{c}
U_{p, 0}^{n+1} \\
0 \\
0 \\
\vdots \\
0 \\
U_{p, N_{y}}^{n+1}
\end{array}\right]}
\end{gathered}
$$

Meanwhile, $V_{p, q}^{n+1}$ is solved iteratively without using Thomas algorithm after getting the values of $U$ and $\theta$. The technique used to find the unknown values in (24) and (25) is similar to the following 
matrix as the general parts of (24) and (25)

$$
\left[\begin{array}{cccccc}
b_{1} & c_{1} & 0 & 0 & \ldots & 0 \\
a_{2} & b_{2} & c_{2} & 0 & \ldots & 0 \\
0 & a_{3} & b_{3} & c_{3} & \ldots & \vdots \\
0 & 0 & \ddots & \ddots & \ddots & 0 \\
\vdots & \vdots & \vdots & a_{N-1} & b_{N-1} & c_{N-1} \\
0 & 0 & \ldots & 0 & a_{N} & b_{N}
\end{array}\right]\left[\begin{array}{c}
v_{1} \\
v_{2} \\
v_{3} \\
\vdots \\
v_{N-1} \\
v_{N}
\end{array}\right]=\left[\begin{array}{c}
d_{1} \\
d_{2} \\
d_{3} \\
\vdots \\
d_{N-1} \\
d_{N}
\end{array}\right]
$$

The matrix (26) is eliminated using Gaussian method, then it reduces to the following matrix

$$
\left[\begin{array}{cccccc}
b^{\prime}{ }_{1} & c_{1} & 0 & 0 & \ldots & 0 \\
0 & b^{\prime}{ }_{2} & c_{2} & 0 & \ldots & 0 \\
0 & 0 & b^{\prime}{ }_{3} & c_{3} & \ldots & \vdots \\
0 & 0 & \ddots & \ddots & \ddots & 0 \\
\vdots & \vdots & \vdots & 0 & b^{\prime}{ }_{N-1} & c_{N-1} \\
0 & 0 & \ldots & 0 & 0 & b_{N}^{\prime}
\end{array}\right]\left[\begin{array}{c}
v_{1} \\
v_{2} \\
v_{3} \\
\vdots \\
v_{N-1} \\
v_{N}
\end{array}\right]=\left[\begin{array}{c}
d^{\prime}{ }_{1} \\
d^{\prime}{ }_{2} \\
d^{\prime}{ }_{3} \\
\vdots \\
d_{N-1}^{\prime} \\
d^{\prime}{ }_{N}
\end{array}\right]
$$

The matrices (26) and (27) can be represented into the following recursive formula to make an easy in iterative processes of numerical simulation

$$
\begin{aligned}
& b_{1}^{\prime}=b_{1}, d^{\prime}{ }_{1}=d_{1}, b_{i}^{\prime}=b_{i}-c_{i-1} \frac{a_{i}}{b^{\prime}{ }_{i-1}} \\
& d_{i}^{\prime}=d_{i}-d^{\prime}{ }_{i-1} \frac{a_{i}}{b^{\prime}{ }_{\mathrm{i}-1}}, i=2,3, \ldots, N
\end{aligned}
$$

and the following backward substitution

$$
v(N)=\frac{d^{\prime}(N)}{b^{\prime}(N)}, v(i)=\frac{d^{\prime}(i)-c(i) v(i+1)}{b^{\prime}(i)}, i=N-1, N-2, \ldots, 2,1
$$

Based on the discretization results by the finite difference method of Crank-Nicolson in (21)(23). We present the following velocity and temperature profiles for different values of angle of inclination $\alpha$, Prandtl number $P_{r}$, and mixed convection parameter $\lambda$

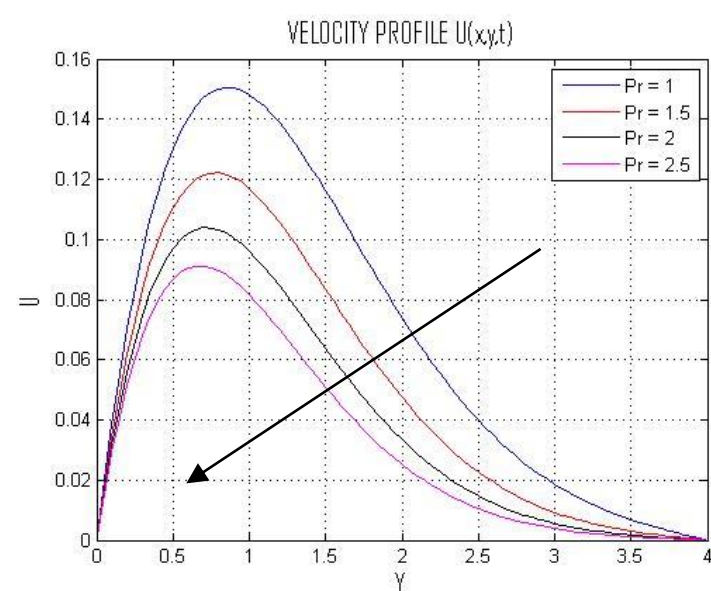

Figure 2. Velocity profile for various Prandtl number $\left(P_{r}\right)$

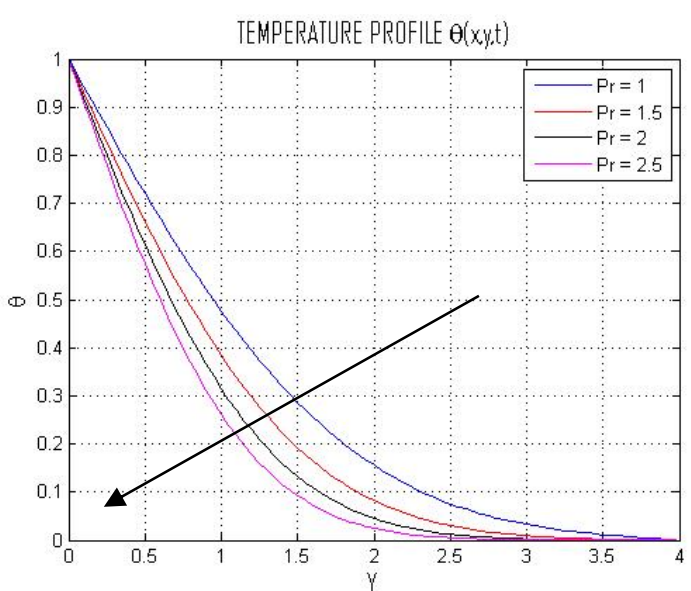

Figure 3. Temperature profile for various Prandtl number $\left(P_{r}\right)$ 


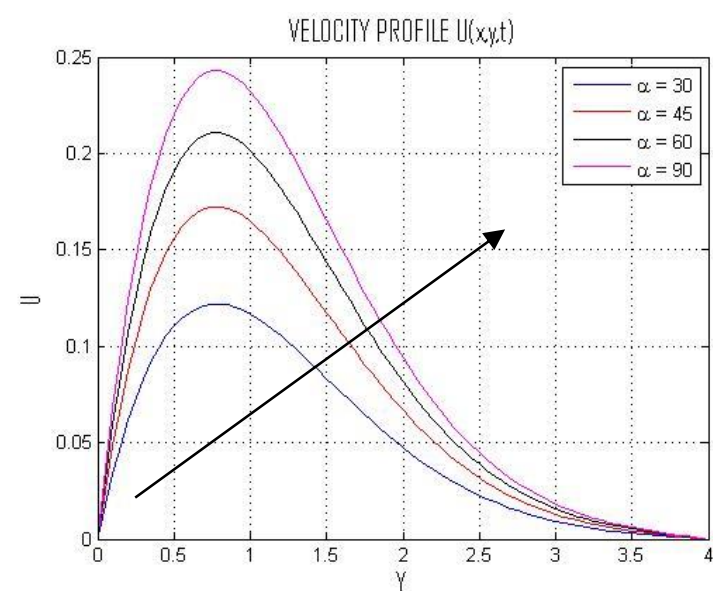

Figure 4. Velocity profile for various angle of inclination $(\alpha)$

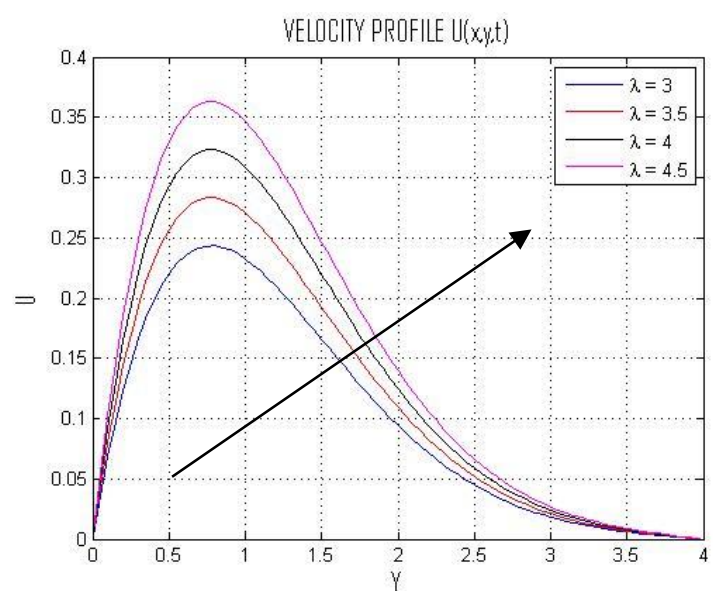

Figure 5. Velocity profile for various mixed convection parameter $(\lambda)$

According to Figure 2, Figure 3, velocity and temperature profiles are more decreased when the different values of Prandtl number are more increased. This is due to the Prandtl number related to thermal diffusivity as written as $P_{r}=\frac{\mu c_{p}}{k}$, which indicates that the more increased Prandtl number is, the more increased thermal diffusivity is. So that, the temperature profile is more decreased. The decreasing of temperature also causes the velocity profile more decreased. Meanwhile, Figure 4 and Figure 5 show that the more increased angle of inclination and mixed convection parameter are, the more increased velocity profile is.

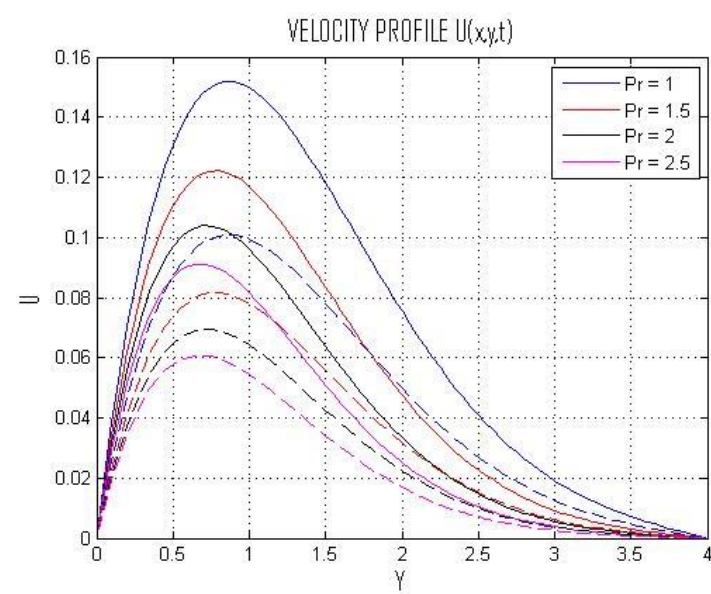

Figure 6. Velocity profile for various Prandtl number $\left(P_{r}\right)$ with $\lambda=1.5$ and $\lambda=0$

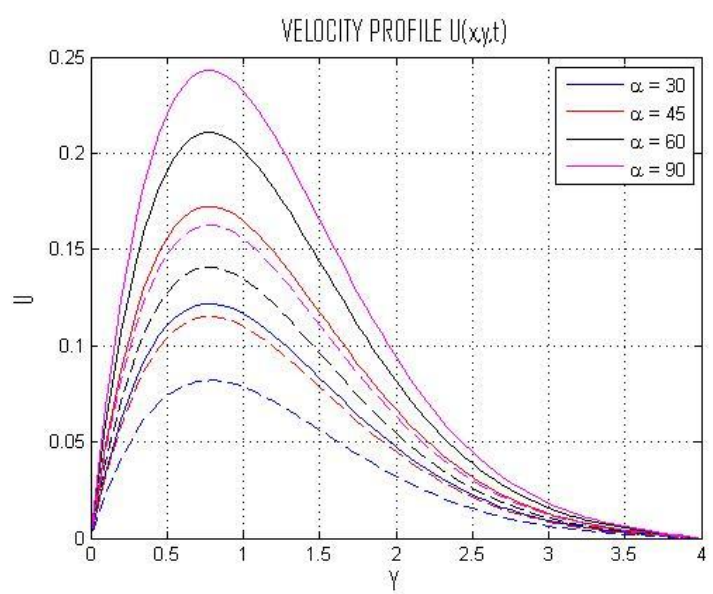

Figure 7. Velocity profile for various angle of inclination $(\alpha)$ with $\lambda=1.5$ and $\lambda=0$

In Figure 6 and Figure 7, the solid line represents the presence of mixed convection flow $(\lambda=$ $1.5)$ and the dashed line represents the absence of mixed convection flow $(\lambda=0)$. Based on those two conditions, the velocity and temperature profiles are same for both (mixed and non-mixed convection flow), which means that the more increased different values of Prandtl number $\left(P_{r}\right)$ are, the more decreased velocity and temperature profiles are and the more increased different values of angle of inclination $(\alpha)$ and mixed convection parameter $(\lambda)$ are, the more decreased velocity profiles are for both (mixed and non-mixed convection flow). Furthermore, the difference for those two conditions are that the velocity profile for the non-mixed case $(\lambda=0)$ is more sloping than the mixed convection flow case $(\lambda=1.5)$. It indicates that there is the external force in the mixed convection flow case $(\lambda=1.5)$, so the velocity of convection flow is higher than the non-mixed case $(\lambda=0)$. 


\section{Conclusions}

Based on the numerical results, then this research can be concluded for the following correlation among the different values of Prandtl number $\left(P_{r}\right)$, angle of inclination $(\alpha)$, and mixed convection parameter $(\lambda)$ with the velocity and temperature profile

1. The more increased different values of Prandtl number $\left(P_{r}\right)$ are, the more decreased velocity and temperature profiles are.

2. The more increased different values of angle of inclination $(\alpha)$ and mixed convection parameter $(\lambda)$ are, the more increased velocity profiles are.

3. The mixed convection flow case $(\lambda=1.5)$ is affected by the external force, so the velocity of convection flow is higher than the non-mixed case $(\lambda=0)$.

\section{Acknowledgement}

The author would like to thank to the reviewer for the valuable comments and suggestions which helped to improve the paper.

\section{References}

[1] N. Amin, R. Nazar, and I. Pop, "On the mixed convection boundary-layer flow about a solid sphere with constant surface temperature," Arabian Journal for Science and Engineering, 27 (2), 117-135, 2002.

[2] R. Nazar, I. Pop and M. Z. Salleh, "Mixed convection boundary layer flow about a solid sphere with newtonian heating," Archives of Mechanics, 62(4), 283-303, 2010.

[3] A. R. M. Kasim, "Convective boundary layer of viscoelastic fluid," Ph.D. Thesis. Malaysia: Universiti Teknologi Malaysia, 2014.

[4] M. Ghani, B. Widodo, and C. Imron, C, "Incompressible and steady mixed convection flow over a sphere," The $1^{\text {st }}$ Young Scientist International Conference of Water Resources Development and Environmental Protection, Malang, Indonesia, 2015.

[5] M. Ghani, and W. Rumite, "Keller-Box scheme to mixed convection flow over a solid sphere with the effect of MHD," MUST: Journal of Mathematics Education, Science and Technology, 6 (1), 97-120, 2021.

[6] B. Widodo, I. Anggriani, D. A. Khalimah, and F. D. S. Zainal, "Unsteady Boundary Layer Magnetohydrodynamics in Micropolar Fluid Past A Sphere," Far East Journal of Mathematical Sciences, 100, 291-299, 2016.

[7] W. A. Khan, Z. H. Khan, and R. Ul Haq, "Flow and heat transfer of ferrofluids over a flat plate with uniform heat flux," The European Physical Journal Plus 130, no. 4, 1-10, 2015.

[8] A. Zeeshan, A. Majeed, and R. Ellahi, "Effect of magnetic dipole on viscous ferro-fluid past a stretching surface with thermal radiation," Journal of Molecular Liquids 215, 549-554, 2016.

[9] R. Jusoh, R. Nazar, and I. Pop, "Magnetohydrodynamic rotating flow and heat transfer of ferrofluid due to an exponentially permeable stretching/shrinking sheet," Journal of Magnetism and Magnetic Materials 465, 365-374, 2018.

[10] M. K. A. Mohamed, N. A. Ismail, N. Hashim, N. M. Shah, and M. Z. Salleh, "MHD slip flow and heat transfer on stagnation point of a magnetite (Fe3O4) ferrofluid towards a stretching sheet with Newtonian heating," CFD Letters 11, no. 1, 17-27, 2019.

[11] S. H. M. Yasin, M. K. A. Mohamed, Z. Ismail, and M. Z. Salleh, "Mathematical solution on MHD stagnation point flow of ferrofluid," In Defect and Diffusion Forum, vol. 399, pp. 3854. Trans Tech Publications Ltd, 2020. 
[12] K. Gangadhar, D. Vijayakumar, A. J. Chamkha, T. Kannan, and G. Sakthivel, "Effects of Newtonian heating and thermal radiation on micropolar ferrofluid flow past a stretching surface: spectral quasi- linearization method," Heat Transfer 49, no. 2, 838-857, 2020.

[13] K. A. Kumar, K. Anantha, N. Sandeep, V. Sugunamma, and I. L. Animasaun, "Effect of irregular heat source/sink on the radiative thin film flow of MHD hybrid ferrofluid," Journal of Thermal Analysis and Calorimetry 139, no. 3, 2145-2153, 2020.

[14] A. M. Rashad, "Impact of anisotropic slip on transient three dimensional MHD flow of ferrofluid over an inclined radiate stretching surface," Journal of the Egyptian Mathematical Society 25, no. 2, 230-237, 2017.

[15] G. Makanda, S. Shaw, and P. Sibanda, "Effects of radiation on MHD free convection of a Casson fluid from a horizontal circular cylinder with partial slip in non-Darcy porous medium with viscous dissipation," Boundary Value Problems 2015, no. 1, 1-14, 2015. 